\title{
Self-collimation and beam splitting in low-index photonic crystals
}

\author{
Aaron F. Matthews *, Steven K. Morrison, Yuri S. Kivshar \\ Nonlinear Physics Centre and Centre for Ultrahigh Bandwidth Devices for Optical Systems (CUDOS), Research School of \\ Physical Sciences and Engineering, Australian National University, Canberra ACT 0200, Australia
}

Received 20 March 2007; received in revised form 17 July 2007; accepted 30 July 2007

\begin{abstract}
We study self-collimation and beam splitting in low-refractive-index photonic crystals created within chalcogenide glass. We propose a beam splitter structure that allows direct experimental verification of photonic-crystal effects at optical wavelengths in a straightforward and definitive manner. The beam splitter provides angular separation of $90^{\circ}$ using a highly compact spatial footprint, thus delivering direct application in highly integrated photonic devices.
\end{abstract}

Crown Copyright (c) 2007 Published by Elsevier B.V. All rights reserved.

PACS: 42.25.Bs; 42.70.Qs; 42.79.Fm; 42.82.Et; 42.87.-d

Keywords: Self-collimation; Chalcogenide glass; Beam splitters; Integrated optics devices

\section{Introduction}

Photonic crystals are periodic structures fabricated through the spatial patterning of dielectrics. Strong dielectric contrasts can lead to photonic band gaps, preventing light with frequencies within the band gap from propagating. Photonic crystals with high-refractive-index contrast have been suggested, fabricated and employed to create mirrors based on the photonic band gap, and in conjunction with defect structures, resonators, waveguides, couplers, and splitters [1-3]. However, the well established lower limit of the refractive-index contrast $(\delta n \simeq 1.31)$ required to produce a spectral band gap in two-dimensional photonic crystals [4] sets a fundamental limit on the materials used to manufacture photonic-crystal devices utilizing the photonic band gap.

Another operating regime for photonic crystals and related structures is the anisotropic, in-band domain, where

\footnotetext{
Corresponding author. Tel.: +61 418794146.

E-mail address: afm124@rsphysee.anu.edu.au (A.F. Matthews).

$U R L$ : http://www.rsphysse.anu.edu.au/nonlinear (A.F. Matthews).
}

substantial diffraction control over propagating Bloch modes occurs for particular crystal geometries and frequency bands. Crystalloid anisotropy produces such phenomenon as negative refraction [5], superprism effects [6] and self-collimation [7]. In turn, these effects can be used for device applications such as flat, sub-wavelength lenses, wavelength-division de-multiplexers, internal reflection bends and beam splitters [8-12]. Previously suggested photonic-crystal beam splitters have been based on photonic crystal-defects [11-13] or have utilized polarization splitting [14]. However, these methods do not provide robust experimental verification of the self-collimation nor photonic crystal-effects. Furthermore, past research into these beam splitters (indeed, most devices utilizing the anisotropic nature of the photonic crystals) has, in general, focused primarily on high-index photonic crystals. Kosaka, Chigrin and Garcia-Pomar have produced seminal work on self-collimation in high-refractive-index photonic crystals $[8,15-17]$. Yet self-collimation within low-refractiveindex materials remains relatively unexplored, with only a few papers considering hybrid structures, such as lowindex materials covered in high-index cladding [18] and 
membrane configurations [19], or low-index self-collimation at microwave wavelengths [20] where fabrication and characterization is far simpler. Dynamic, nonlinear control over the material properties of optical devices is essential to the creation of highly functional optical systems. Useable nonlinearities for small-scale optical devices require nontraditional materials that, in general, have low-refractiveindex values. For example, traditional high index materials such as silicon and gallium arsenide have typical nonlinearities of $n_{2}=0.45 \times 10^{-13}\left(\mathrm{~cm}^{2} / \mathrm{W}\right)$ and $n_{2}=1.59 \times 10^{-13}$ $\left(\mathrm{cm}^{2} / \mathrm{W}\right)[21]$, respectively. Conversely, chalcogenides possess nonlinear coefficients hundreds of times greater [22], but at the expense of much lower indices of refraction, resulting in only minor or partial directional band gaps in photonic crystals [23].

To obtain dynamic functionality also requires stringent control over the geometry of photonic crystal, necessitating advanced fabrication techniques. Chalcogenide photoniccrystal fibers have been demonstrated by a large number of groups [24], and this technology has been adapted to create one-dimensional Bragg gratings [25,26] and simple stacked chalcogenide photonic crystals [27-33]. However, difficulties still exist in the fabrication of functional twodimensional and planar photonic crystals, and greater difficulties occur in the experimental verification of these structures. In recent years, commercially available fabrication equipment, most notably focused ion beam (FIB) systems, have improved considerably, allowing improved fabrication of planar photonic crystals within chalcogenide glasses. Yet, fabrication impediments still prevent the integrated coupling of light into photonic crystals, obscuring the confirmation of distinctly photonic-crystal-based effects [23].

In this paper, we analyze the self-collimation effect in low-refractive-index photonic crystals with two-dimensional geometry, and demonstrate significant diffraction control over propagating Bloch modes within these lowindex structures at optical wavelengths. In addition, we detail a robust method to experimentally verify the basic properties of photonic crystals fabricated in low-index materials utilizing only the self-collimation effect. In doing so, we give a specific example of the method applied to chalcogenide-glass photonic crystals that exhibits significant experimental difficulties to confirm distinctly photonic-crystal-based effects [23]. Finally, we discuss the concept of a novel photonic-crystal beam splitter that delivers high-level integration density in a wide range of materials.

\section{Self-collimation in chalcogenide photonic crystals}

Self-collimation is a corollary of the anisotropic nature of photonic crystals that confines wave vectors of propagating modes within the self-collimation band to one direction, preventing diffraction. From the well-known relationship $v_{\mathrm{g}}=\partial \omega / \partial \mathbf{k}$ (where $v_{\mathrm{g}}$ is the group velocity, equivalent to the average energy flow, and $\omega$ and $\mathbf{k}$ are the frequency and wave vector respectively) the propagation direction of light within a photonic crystal can be determined. This can be viewed graphically using dispersion surfaces or, the two-dimensional equivalent, iso-frequency contours, as illustrated in Fig. 1d. Within the figure, the light propagation direction is defined by the projection of the dispersion surface gradient onto the wave vector plane. Accordingly, regions where the iso-frequency contours are straight cause all wave vectors, within the angular space and frequency range, to be guided in a parallel direction, which through symmetry, corresponds to the propagation direction through the crystal.

We study self-collimation in both two-dimensional and three-dimensional planar chalcogenide photonic crystals formed using a square lattice of air holes. The structures considered have a normalized lattice period of $a$ corresponding to a period of $403 \mathrm{~nm}$ for light with a $1550 \mathrm{~nm}$ wavelength, and a hole radius of $0.43 a$, equivalent to $173.29 \mathrm{~nm}$. The TE polarized (magnetic field parallel to the axis of the holes) refractive-index of the chalcogenide was experimentally determined to be 2.548 [34], exhibiting negligible material losses [35], and is employed for both photonic-crystal geometries. Fig. 1a and b illustrate schematically the geometry of the crystals. We define the height of the planar structure using typical experimental aspect ratios achieved using FIB fabrication systems. This aspect ratio (substrate height-to-hole radius) is generally limited to $1: 1$ because of re-deposition of chalcogenide on the perforation walls as the substrate height increases. Consequently, we take the substrate height to be $0.43 a$ or $173.29 \mathrm{~nm}$. Numerical modeling of the structures is performed using both the plane wave expansion method $[36,37]$ and the finite-difference time-domain method $[38,39]$.

The band diagram for both the two-dimensional and three-dimensional planar structure are shown in Fig. 1c, along with the light cone that defines the region where light will not be confined to the planar structure by index-confinement. A band gap exists for the two-dimensional structure between $\omega a / 2 \pi c=0.301$ and 0.314 , however, no band gap exists for the planar structure, highlighting the limitations of chalcogenide photonic crystals with planar geometry.

Importantly however, self-collimation is possible over a large wave vector range within both two-dimensional and planar chalcogenide photonic crystals, as illustrated by the long, straight iso-frequency contour seen in Fig. 1d and e, within the approximate frequency range of $\omega a / 2 \pi c=0.25$ to 0.28 . The effect of the three-dimensional planar geometry is to increase the frequency range of the dispersion surface. This occurs due to a finite localization of the fields outside the slab; effectively further reducing the refractive index experienced by the propagating modes, pushing their frequencies higher. Significantly, the lowrefractive index of the chalcogenide substrate, combined with the frequency lifting effect of the planar geometry 
a

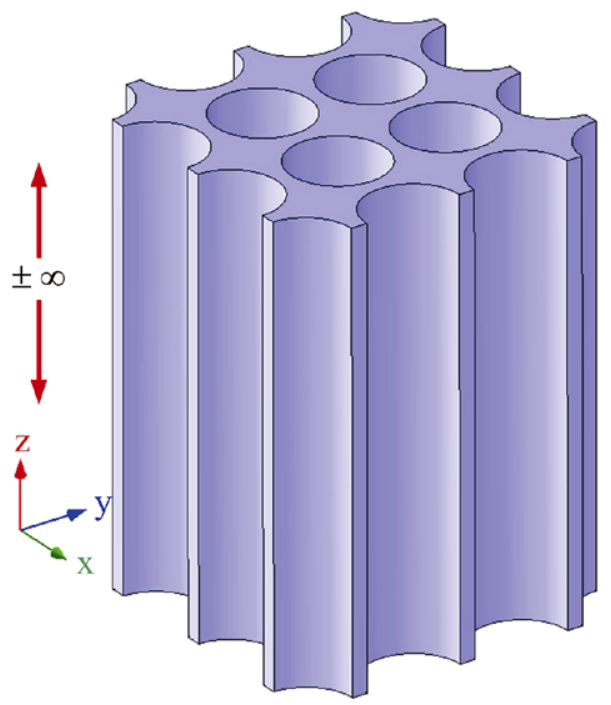

b

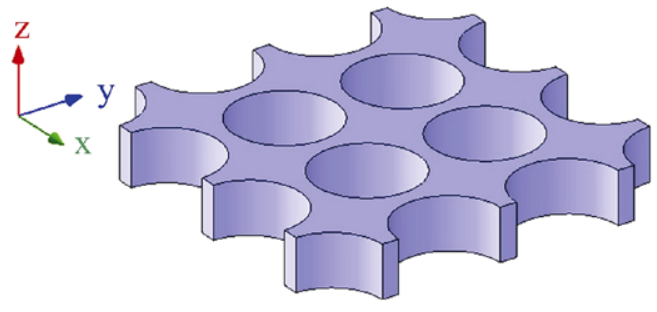

C

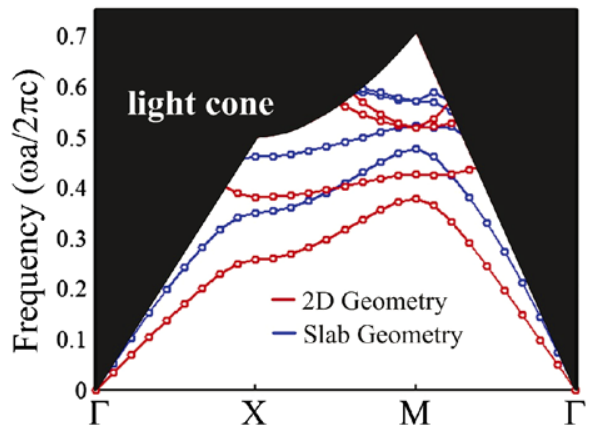

d
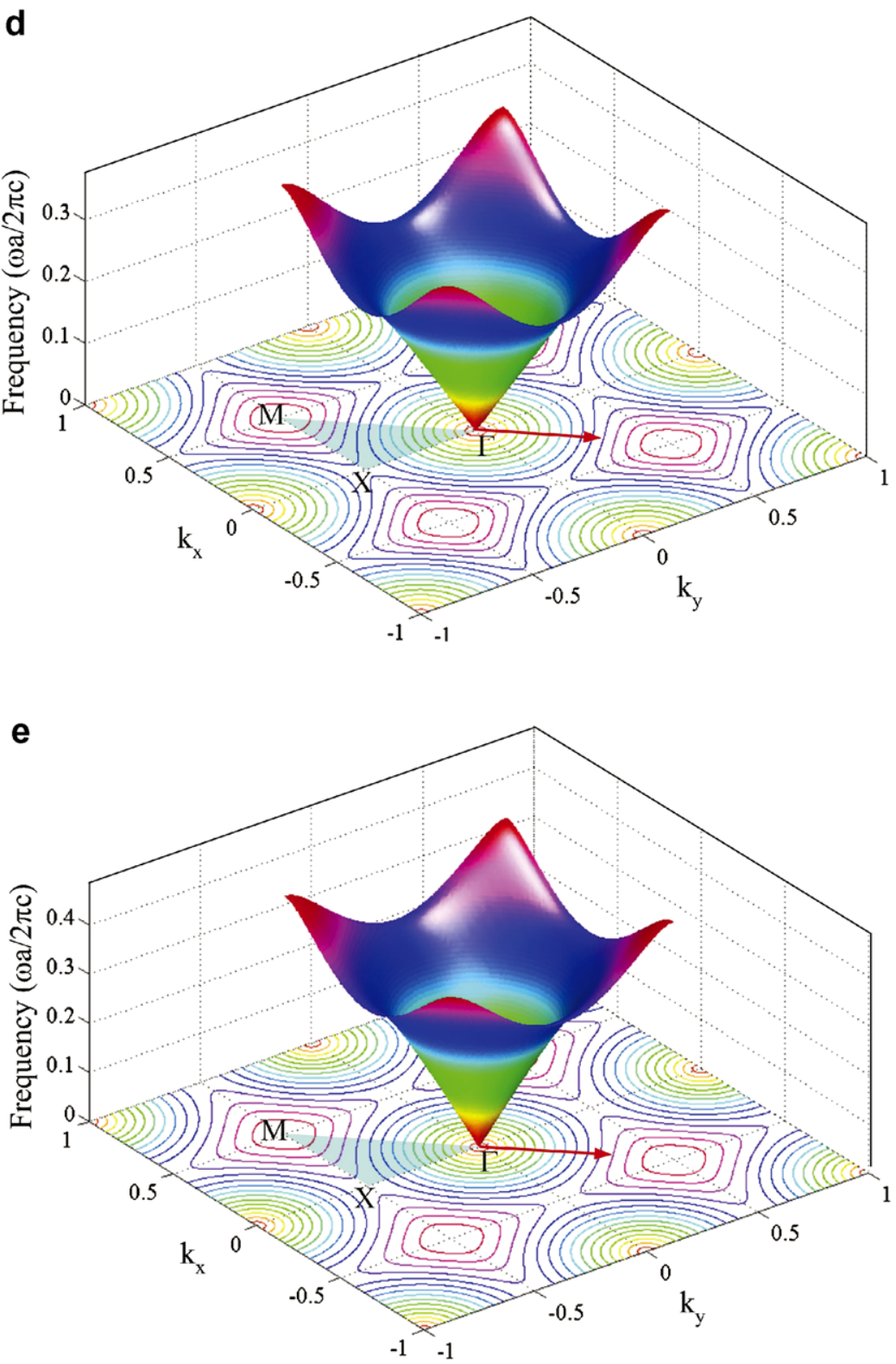

Fig. 1. Self-collimation systems: (a and b) schematic representations of the two- and three-dimensional photonic crystals; (c) band diagram for both photonic crystals highlighting the light cone limits of the planar structure; ( $\mathrm{d}$ and e) dispersion surfaces and iso-frequency contours for two photonic crystals illustrating the minor effect of the planar structure on the dispersion surface.

does not prohibit self-collimation, presenting a robust, functional mechanism for chalcogenide photonic crystals created in planar substrates. The effect of these self-collimation dispersion surfaces within an infinite photonic crystal is demonstrated in Fig. 2, where light is prevented from propagating - without a band gap - in directions non-perpendicular to the self-collimating dispersion surfaces.

We analyze the diffractive nature of light within chalcogenide glass material and the chalcogenide based photonic crystals in the region of the self-collimation frequency. Fig. 3 illustrates the symmetry-invariant diffractive nature of light propagation in an isotropic medium of chalcogenide glass, for several source frequencies, demonstrating distinct diffraction along the propagating direction. Also shown in Fig. 3 is the strong confinement of light due to the flat, self-collimation iso-frequencies contours of the photonic crystals. The periodic patterning from the photonic crystals also gives rise to a wave vector mismatch between the iso-frequency contours of the unperturbed chalcogenide glass - the inner blue circles of the iso-frequency plots - and the iso-frequency contours of the photonic crystals. In turn, this inhibits light coupling into the photonic crystals for frequencies above the self-collimation band.

From an experimental prospective, Fig. 3 illustrates the difficulty associated with conclusive verification of self-collimation within photonic crystals of fabricable 


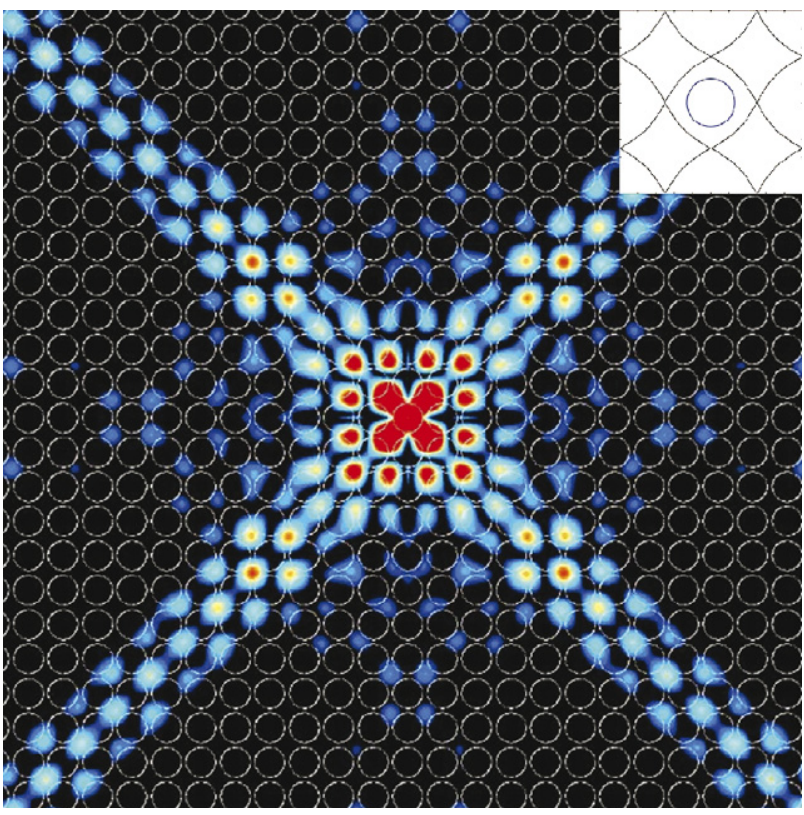

Fig. 2. Dispersive control of a dipole source in an infinite, low-index photonic crystal; including suppression of light in some directions without a directional band gap. Inset: Iso-frequency contours at the dipole source frequency. size. As diffraction length is proportional to the input beam width, an experimental setup requires either a small, tightly focused input beam and minute detector, or a very large photonic crystals. Qualitatively, we examine the required experimental conditions for a Gaussian input beam using $w(z)=w_{0}\left[1+\left(2 z / k w_{0}^{2}\right)\right]^{1 / 2}$, where $w(z)$ is the $1 / e^{2}$ irradiance contour radius after the beam has traveled a distance $z, w_{o}$ is the initial $1 / e^{2}$ irradiance contour radius or beam waist, and $k$ is the self-collimation wave vector which can be determined from the iso-frequency-contour figure. Using an experimentally attainable Gaussian input beam waist of $100 \mu \mathrm{m}$, in conjunction with a detector with a spatial resolution of $30 \mu \mathrm{m}$, requires a photonic crystal with a minimum length of $4.28 \mathrm{~cm}$. A photonic crystal of this size presents experimental complications in itself, such as the crystal's inability to provide mechanical strength to support its own weight, along with warping and twisting of a planar membrane over such comparatively large expanses. These spatial limitation also exist for alternative methods that visualize the out of plane radiation pattern of the photonic crystal [40].

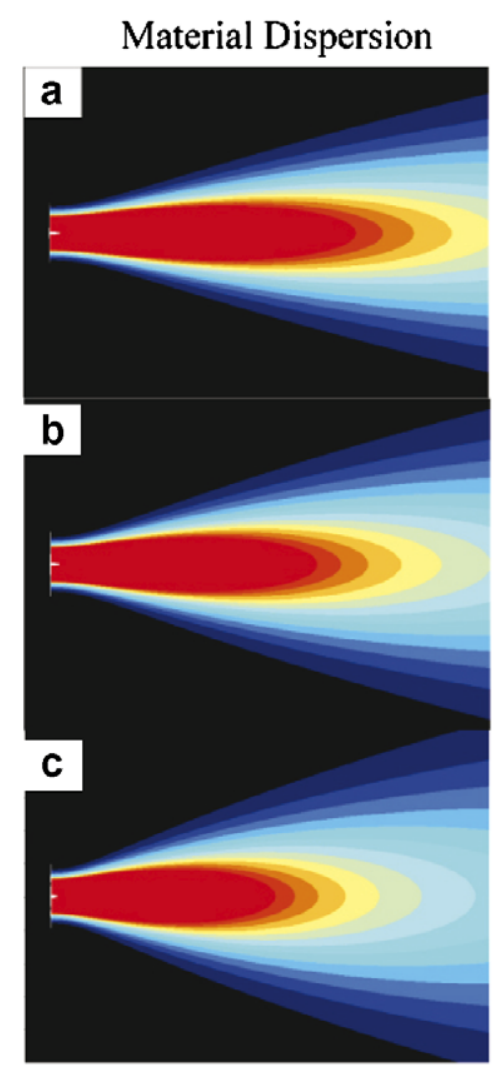

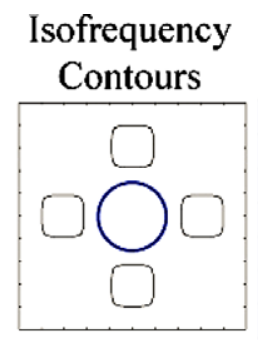
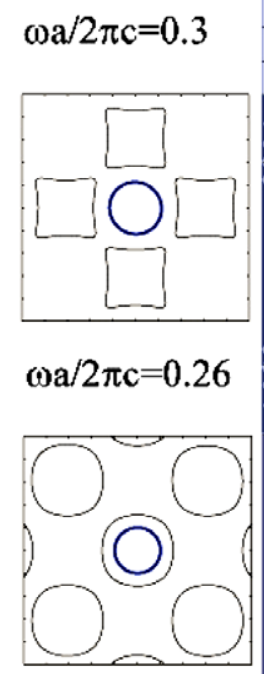

$\omega \mathrm{a} / 2 \pi \mathrm{c}=0.22$

\section{Transmitted}

Intensity

Poynting Vector

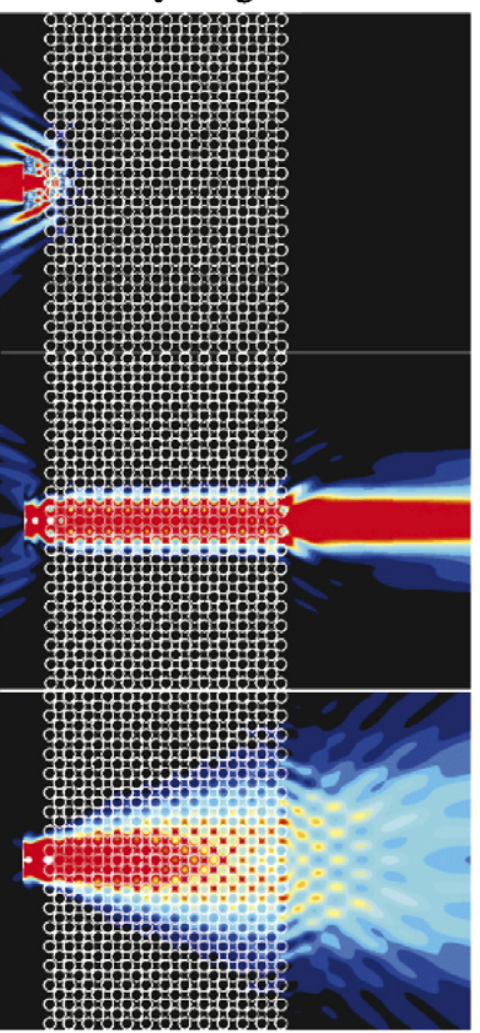

Fig. 3. Poynting vector of the chalcogenide glass only; the iso-frequency contours for both the chalcogenide glass and the chalcogenide based photonic crystals; the output transmitted intensity from the photonic crystals, and Poynting vector of the photonic crystals for: (a) $\omega a / 2 \pi c=0.3$; (b) $\omega a / 2 \pi c=0.26$; and, (c) $\omega a / 2 \pi c=0.22$. Blue circles in the iso-frequency contours indicate the contours of the isotropic chalcogenide glass (For interpretation of the references in color in this figure legend, the reader is referred to the web version of this article). 


\section{Self-collimated beaming splitter}

To enable experimental verification of self-collimation at optical wavelengths, we propose a novel beam splitter that divides an incident beam into two transmitted beams, separated by an angle of $90^{\circ}$, under self-collimation conditions. Splitting of the incident beam is achieved by rotating the crystal by $45^{\circ}$ thereby translating the self-collimation surface from the forward direction into two surfaces along the $45^{\circ}$ directions. This produces an aggregate triangular shaped photonic crystal, as illustrated in Fig. 4. The purpose of the surface cuts perpendicular to the propagation direction of the two beams is to provide a homogeneous transition out of the beam splitter, preventing disruption to the transmitted wave fronts and consequently the beams themselves.

As self-collimation occurs in the $\Gamma-M$ direction of the crystal, an incident beam perpendicular to the $\Gamma-X$ surface will split with increased power density conveyed in the $\Gamma-M$ directions, but this will also be accompanied by a large amount of reflection at the $\Gamma-X$ surface. To produce two highly localized output beams along the $\Gamma-M$ directions we decrease the input frequency, until the onset of the directional band gap in the $\Gamma-X$ direction. Precise conditions for self-collimation are no longer met, but the output beams experience negligible diffraction due to the minor divergence of the iso-frequency contours and the small propagation distance through the crystal. However, the larger iso-frequency contours collect more wave vectors, and hence power, while the sharp intersection angle of the equifrequency surfaces prohibits propagation in the $\Gamma-X$ direction, producing a strong null, enabling the discernible detection of the two output beams. This condition is illustrated in Fig. 4b, which shows the output intensity and power density, for positive confirmation of self-collimation. Fig. 4a and c, illustrate the directly measurable conditions away from the self-collimation operating regime, which in combination with the selfcollimation condition provides mutually conclusive evidence of the self-collimation effect.

We also suggest an alternative measurement technique that does not require a tunable laser source, but instead employs the beam splitter's sensitivity to the input incident angle. By changing the incident angle, power distribution to the two output beams can be switched completely into one arm of the splitter. This angular dependent, intensity switching is shown in Fig. 5 where the incident angle is varied up to $45^{\circ}$ with respect to the normal.

As the incident angle moves away from the normal, power is still directed into both output arms of the splitter. However, the division of power is now a function of the angular spread of wave vectors that define the input beam. Further increases to the incident angle directs more wave vectors onto one of the symmetric self-collimation regions of the iso-frequency contours, with a diminishing quantity falling on the other self-collimation direction,
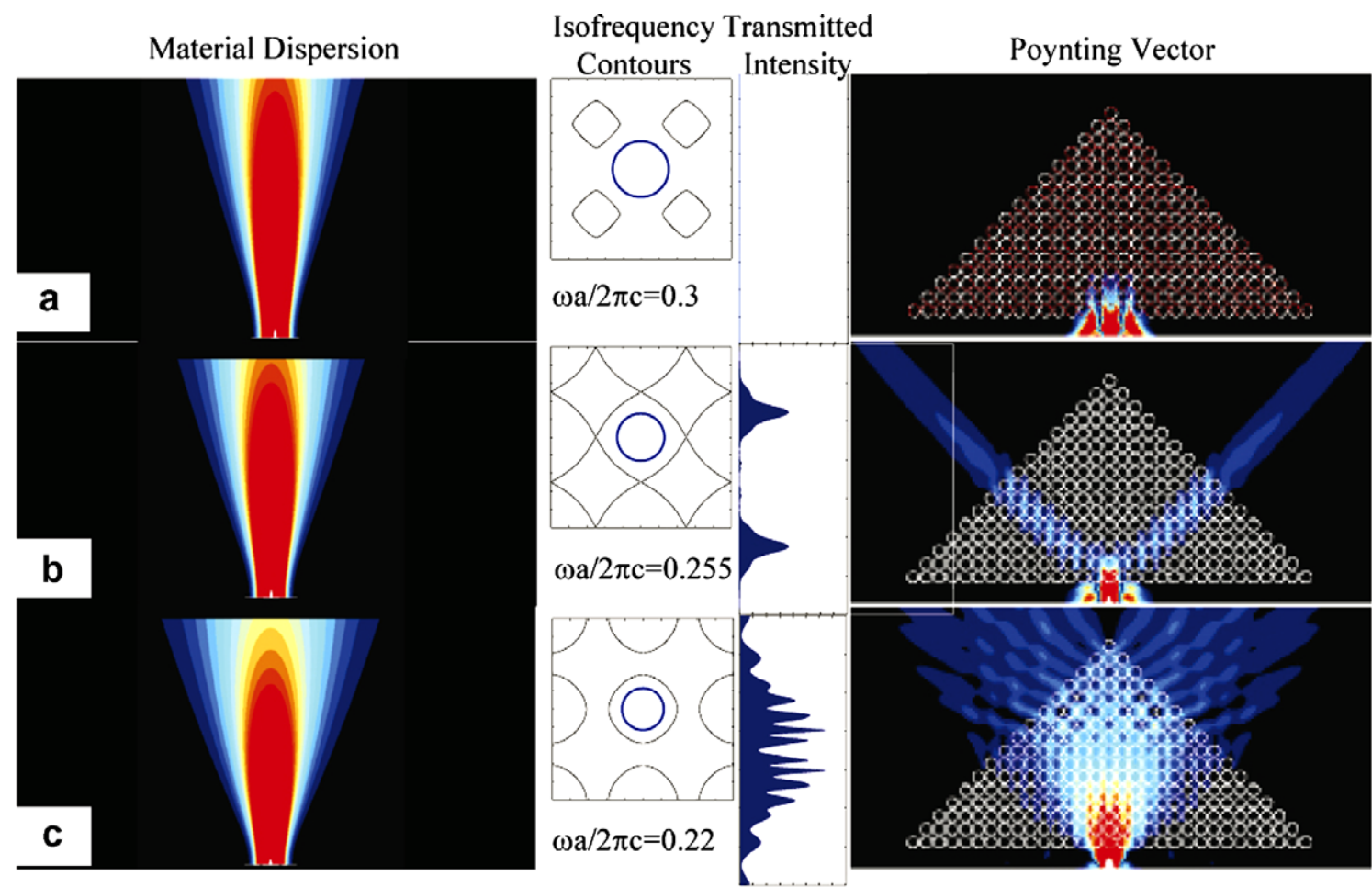

Fig. 4. IFC, output transmitted intensity and Pointing vector of the photonic-crystal beam splitter for: (a) $\omega a / 2 \pi c=0.3$; (b) $\omega a / 2 \pi c=0.255$; and, (c) $\omega a$ / $2 \pi c=0.22$. Again, the blue circles in iso-frequency contours plots indicate the contours of isotropic chalcogenide glass (For interpretation of the references in color in this figure legend, the reader is referred to the web version of this article). 

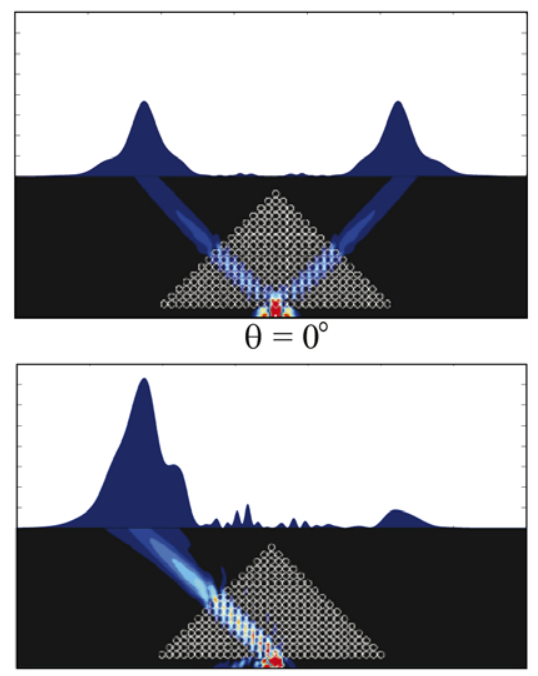

$\theta=30^{\circ}$

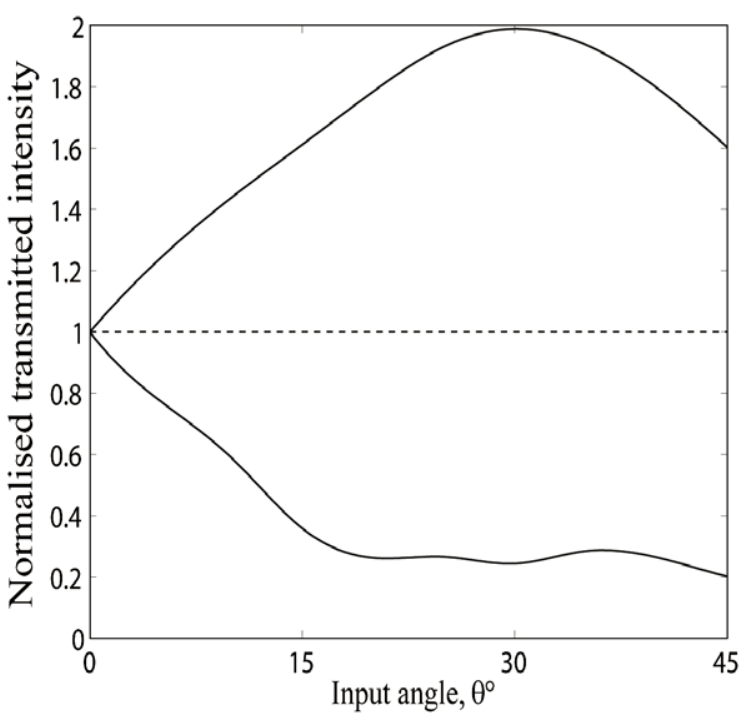

Fig. 5. Left: Poynting vector and transmitted intensity from the beam splitter for perpendicular incidence and $30^{\circ}$ angular incidence. Right: The relative power in each arm of the beam splitter normalized to the normal-input beam for changing incidence angle.

giving rise to the functional switching of the output power. This alternative measurement methodology again provides a strong, simple technique for the verification of the self-collimation effect within low-index photonic crystals.

\section{Conclusions}

We have analyzed the specific properties of the self-collimation effect in low-index photonic crystals created within chalcogenide glass and demonstrated that this effect can be observed even in planar photonic-crystal geometries. We have suggested a novel type of beam splitter structure based upon self-collimation that would allow experimental verification of the photonic-crystal effects at optical wavelengths and within low-index materials, in a straightforward and definitive manner. This self-collimation-based beam splitter provides a large angular separation of $90^{\circ}$ between the output beams, using a small spatial footprint. The large beam separation, in combination with a small device size, may provide significant advantages for optical integration.

\section{Acknowledgements}

The authors thank Dr. Guangyong Zhou and Dr. Steve Madden for useful discussions. This work was supported by the Australian Research Council through the Centers of Excellence Program.

\section{References}

[1] Y. Fink, J.N. Winn, S. Fan, C. Chen, J. Michel, J.D. Joannopoulos, E.L. Thomas, Science 282 (1998) 1679.
[2] J.D. Joannopoulos, P.R. Villeneuve, S. Fan, Nature 386 (1997) 143.

[3] M. Bayindir, E. Ozbay, B. Temelkuran, M.M. Sigalas, C.M Soukoulis, R. Biswas, K.M. Ho, Phys. Rev. B 63 (2001) 081107.

[4] A. Matthews, X.-H. Wang, Yu. S. Kivshar, M. Gu, Appl. Phys. B 81 (2005) 189.

[5] M. Notomi, Phys. Rev. B 62 (2000) 10696.

[6] S.-Y. Lin, V.M. Hietala, L. Wang, E.D. Jones, Opt. Lett. 21 (1996) 1771.

[7] P. Rakich, M. Dahlem, S. Tandon, M. Ibanescu, M. Soljacic, G. Petrich, J. Joannopoulos, L. Kolodziejski, E. Ippen, Nature Mat. 5 (2006) 93.

[8] J. Garcia-Pomar, M. Nieto-Vesperinas, Opt. Express 13 (2005) 7997.

[9] C. Luo, S.G. Johnson, J.D. Joannopoulos, J.B. Pendry, Phys. Rev. B 65 (2002) 201104.

[10] B. Gralak, S. Enoch, G. Tayeb, J. Opt. Soc. Am. A 17 (2000) 1012.

[11] X. Yu, S. Fan, Appl. Phys. Lett. 83 (2003) 3251.

[12] S. Shi, A. Sharkawy, C. Chen, D.M. Pustai, D.W. Prather, Opt. Lett. 29 (2006) 617.

[13] D.M. Pustai, S. Shi, C. Chen, A. Sharkawy, D.W. Prather, Opt Express 12 (2004) 1823

[14] V. Zabelin, L. Dunbar, N. Le Thomas, R. Houdre, M. Kotlyar, L. O'Faolain, T. Krauss, Opt. Lett. 32 (2007) 530.

[15] H. Kosaka, T. Kawashima, A. Tomita, M. Notomi, T. Tamamura, T. Sato, S. Kawakami, Phys. Rev. B 58 (1998) R10096-9.

[16] H. Kosaka, T. Kawashima, A. Tomita, M. Notomi, T. Tamamura, T. Sato, S. Kawakami, Appl. Phys. Lett. 74 (1999) 1212.

[17] D. Chigrin, S. Enoch, C. Sotomayor Torres, G. Tayeb, Opt. Express 11 (2003) 1203.

[18] M. Augustin, R. Iliew, C. Etrich, D. Schelle, H.-J. Fuchs, U. Peschel, S. Nolte, E.-B. Kley, F. Lederer, A. Tünnermann, Appl. Phys. B 81 (2005) 313 .

[19] L. Wu, M. Mazilu, J.-F. Gallet, T.F. Krauss, Phot. Nanostruct. Fund. Appl. 1 (2003) 31.

[20] Z. Lu, C. Schuetz, S. Shi, C. Chen, G. Behrmann, D. Prather, IEEE Trans. Microw. Theory Tech. 53 (2005) 1362.

[21] M. Dinu, F. Quochi, H. Garcia, Appl. Phys. Lett. 82 (2003) 2954.

[22] A. Zakery, S.R. Elliot, J. Non-Cryst. Solids 330 (2003) 1, and references therein.

[23] C. Grillet, C. Smith, D. Fleeman, S. Madden, B. Luther-Davies, E. Magi, D. Moss, B. Eggleton, Opt. Express 14 (2006) 1070.

[24] See for example P.St.J. Russell, Science 299 (2003) 358, and reference therein. 
[25] C. Becker, M. Wegener, S. Wong, G. von Freymann, Appl. Phys. Lett. 89 (2006) 131122

[26] H.-Y. Lee, T. Yao, J. Vac. Sci. Technol. B 20 (2002) 2017.

[27] B. Juarez, S. Rubio, J. Sanchez-Dehesa, C. Lopez, Adv. Mater. 14 (2002) 1486.

[28] S. Wong, M. Deubel, F. Pérez-Willard, S. John, G. Ozin, M. Wegener, G. von Freymann, Adv. Mater. 18 (2006) 265.

[29] A. Feigel, Z. Kotler, B. Sfeza, A. Arsh, M. Klebanov, V. Lyubin, Appl. Phys. Lett. 78 (2000) 3221.

[30] V.N. Astratov, A.M. Adawi, M.S. Skolnick, V.K. Tikhomirov, V. Lyubin, D.G. Lidzey, M. Ariu, A.L. Reynolds, Appl. Phys. Lett. 78 (2001) 4094

[31] A. Feigel, M. Veinger, B. Sfez, A. Arsh, M. Klebanov, V. Lyubin, Appl. Phys. Lett. 83 (2003) 4480.
[32] U. Jeong, P.H.C. Camargo, Y. Lee, Y. Xia, J. Mater. Chem. 16 (2006) 3893.

[33] V. Lyubin, M. Klebanov, A. Feigel, B. Sfez, Thin Solid Films 459 (2004) 183

[34] Steve Madden (personal communications).

[35] Loss mechanisms based on coupling of light into non self-collimated directions are modelled, but result in no significant issues of device operation or experimental verification.

[36] K. Ho, C. Chan, C. Soukoulis, Phys. Rev. Lett. 65 (1990) 3152.

[37] S. Johnson, J.D. Joannopoulos, Opt. Express 8 (2001) 173.

[38] K.S. Yee, IEEE Trans. Antenn. Propag. AP-14 (1966) 302.

[39] Optiwave systems Inc. <http://www.optiwave.com>.

[40] B. Lombardet, L. Dunbar, R. Ferrini, R. Houdre, F. Robin, J. Appl. Phys. 99 (2006). 